\title{
Erratum
}

\section{Genomic medicine: challenges and opportunities for physicians}

\author{
H Burton, T Cole and AM Lucassen
}

Clinical Medicine 2012;5:416-9.

Errors were introduced to Table 1 in this article during typesetting. The 'Genetically predisposed subgroup' cell of the breast cancer row should read 'About $5 \%$ of cancers will be largely due to inheritance of a mutation in BRCA1 or BRCA2', and the 'Importance of molecular diagnosis in management' cell of breast cancer should read 'Further preventive management for breast cancer and/or ovarian cancer (eg mammography or MRI; prophylactic surgery of breasts and /or ovaries)'. The 'Genetically predisposed subgroup' cell of the bowel cancer row should read 'About 5\% of all cancers will be due, in large part, to inheritance of a mutated gene: eg familial polyposis, Lynch syndrome or MutYH polyposis' and the 'Importance of molecular diagnosis in management' cell for bowel cancer should read 'Consideration of other at-risk organs (eg uterus or ovaries in Lynch syndrome)'.

The corrected table is printed here.

Table 1. Common conditions and genetically predisposed subgroups.

Condition/disease/

presentation to mainstream

clinical specialist

Arrhythmia/syncope/SCD

Breast cancer

Diabetes

High cholesterol/premature CHD

Obesity

Congenital deafness
Genetically predisposed

subgroup

- Ion channelopathies (eg LQT syndrome)

- About $5 \%$ of cancers will be largely due to inheritance of a mutation in BRCA1 or BRCA2
Underlying genetic variation

- Several different genes related to LQT syndrome that, if mutated, predispose to arrhythmias and SCD

- BRCA1 and BRCA2 mutations

- $A P C$ gene-related familial polyposis

- Mismatch repair genes (eg MSH2, Mlh1)

- Mutations in MYH (recessive inheritance)

- Mutations in MODY gene

Maturity-onset diabetes in young people

- Familial

hypercholesterolaemia

- Young age of onset of high cholesterol

- Severe, early-onset obesity that may be associated with severe insulin resistance and other endocrinological disturbances

- Usher's syndrome regulation of appetite) and
- LDL receptor gene or apolipoprotein B gene

- Genes involved in production of leptin, which is involved in downstream pathways

- At least 11 different genetic loci; recessive condition, so both parents need to be carriers

\section{Importance of molecular diagnosis in management*}

- Preventive treatments such as $\beta$ blockers

- Implantable defibrillator

- Further preventive management for breast cancer and/or ovarian cancer (eg mammography or MRI; prophylactic surgery of breasts and /or ovaries)

- Colonoscopic surveillance and removal of adenomas

- Consideration of other at-risk organs (eg uterus or ovaries in Lynch syndrome)

- Aspirin to reduce incidence of adenomas

- Respond to treatment with sulphonylurea, so insulin treatment may not be necessary

- Treatment with statins, using highintensity statins as necessary

- Stringent restriction of access to food in patients with monogenic obesity leptin treatment in some

- Recognition of Usher's syndrome is important, as the child will also lose vision and so should have early intervention to enhance communication (eg cochlear implantation)

$\mathrm{CHD}=$ coronary heart disease; $\mathrm{LDL}=$ low-density lipoprotein; $\mathrm{LQT}=$ long QT; MRI = magnetic resonance imaging; SCD = sudden cardiac death.

*Includes consideration and communication of risk to family members or future family members. Molecular diagnosis allows cascade testing to take place to identify affected individuals and can reassure those who have not inherited the familial predisposition that they do not need interventions. 